\title{
Scenarios for the Spread of COVID-19 in Manaus, Northern Brazil
}

\author{
SANDRO BITAR \& WILHELM ALEXANDER STEINMETZ
}

\begin{abstract}
We use a compartmental model to project scenarios for the spread of COVID-19 in the city of Manaus, with different degrees of social distancing and isolation of infectious individuals. In a scenario without any containment measures, our projections point to more than 50,000 (simultaneously) infectious individuals before the end of April 2020. Only in a scenario with strict and extensive social distancing (lockdown), implemented and observed as of March $23^{\text {rd }}, 2020$, would this number be less than 50,000 in the first half of June. Furthermore, an increase in the number of tests or in the collective awareness among the population regarding the importance of strict distancing measures would have only had a significant impact if implemented before the end of March.
\end{abstract}

Key words: compartment model, COVID-19, epidemiology, infectious diseases.

\section{INTRODUCTION}

On December $31^{\text {st }}, 2019$, the World Health Organization (WHO 2020) was informed of cases of pneumonia of unidentified origin in the city of Wuhan, Province of Hubei, China. In the following weeks, the etiological agent was identified as a new coronavirus, subsequently called SARS-CoV-2, and the disease caused by it denominated COVID-19. In the first months of the year 2020, cases of COVID-19 were identified in several countries around the world, and on March $11^{\text {th }}, 2020$, the outbreak of this disease was declared a pandemic by the WHO.

On March $13^{\text {th }}$, the first case of COVID-19 was reported in the State of Amazonas, Brazil and on March $23^{\text {rd }}, 2020$, the Governor of the State decreed a public health emergency and determined social distancing measures. On April $6^{\text {th }}, 2020,532$ cases of the disease were registered, including 473 in Manaus, the capital of the State of Amazonas, according to the Fundação de Vigilância em Saúde of the State of Amazonas (FVS-AM 2020). In the last few months, studies have been carried out on the possible future spread of the virus, such as the reports by the Imperial College in London (2020), including a study on the spread in the metropolitan region of São Paulo by Rocha Filho et al. (2020). In this work we study possible scenarios for the spread of COVID-19 in Manaus, using a SIQR compartment model. Most compartment models used in this pandemic (Prem et al. 2020, Castilho et al. 2020) have explored age-structuring as in Inaba 2006. We decided not to work with such a structuring to focus solely on the effect of social distancing in the general population and on the isolation of infectious individuals, removed from the population of individuals capable of transmitting the disease. The study of Castilho et al. (2020) also considers the effect of the quarantine of infected individuals. In our work, however, we also try to model the effect of spontaneous and voluntary self-isolation (due to a better 
understanding of the risks of the disease for the general population) by individuals that suspect having been infected.

There is currently only little reliable data available on this disease and still less data specific to the local Brazilian scenario. We work with estimates of parameters according to global data on the disease. We analyse scenarios for the spread of the disease with different degrees of social distancing measures and isolation of part of the population.

\section{BASIC SCHEME OF THE MODEL}

Since COVID-19 is a new disease, there is still no clear scientific data on whether infected and recovered individuals develop immunity in the short, medium or long term. There are reports of reinfections in Japan, but scientific implications of these are not clear enough and Bao et al. (2020) showed in a study that reinfection in rhesus monkeys with SARS-CoV-2 was improbable. Therefore we choose to assume that reinfection is unlikely, pointing out that the choice of admitting reinfections or not in the model has consequences especially in the long term. Inferences about longer term are difficult because they depend on future actions of public agents. We will assume the absence of a vaccine or its manipulation in the medium and short term.

The mathematical model that we use to project the scenarios can be described by the flow chart in Figure 1, with compartments corresponding to different groups of the population of Manaus - the population of individuals susceptible to COVID-19 is denoted by $S(t)$, that of the infectives by I( $t)$, that of the isolated by $Q(t)$ and the part of the population that has recovered from COVID-19 is denoted by $R(t)$.

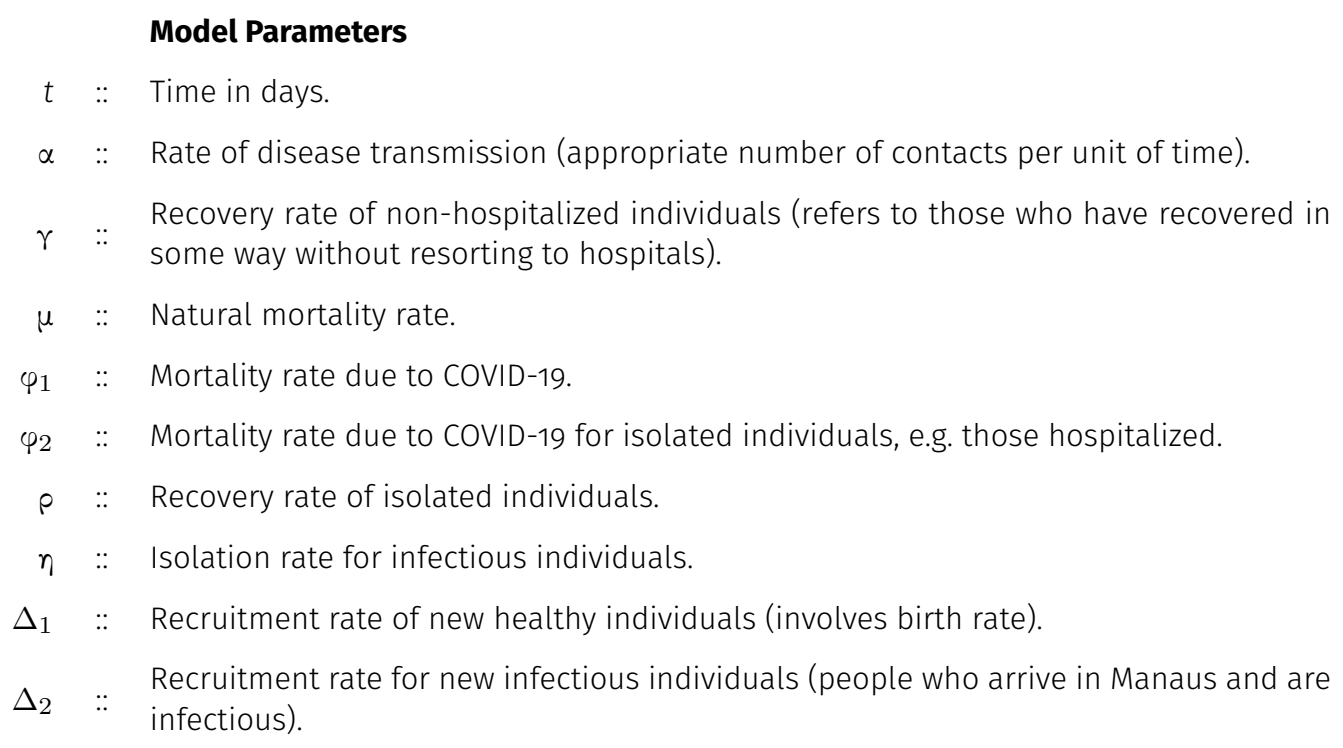

\section{Some Hypotheses}

As COVID-19 is a new disease with no indication of the existence of immunity in the population, we initially assume that the entire population is susceptible, apart from a small number of infectious 


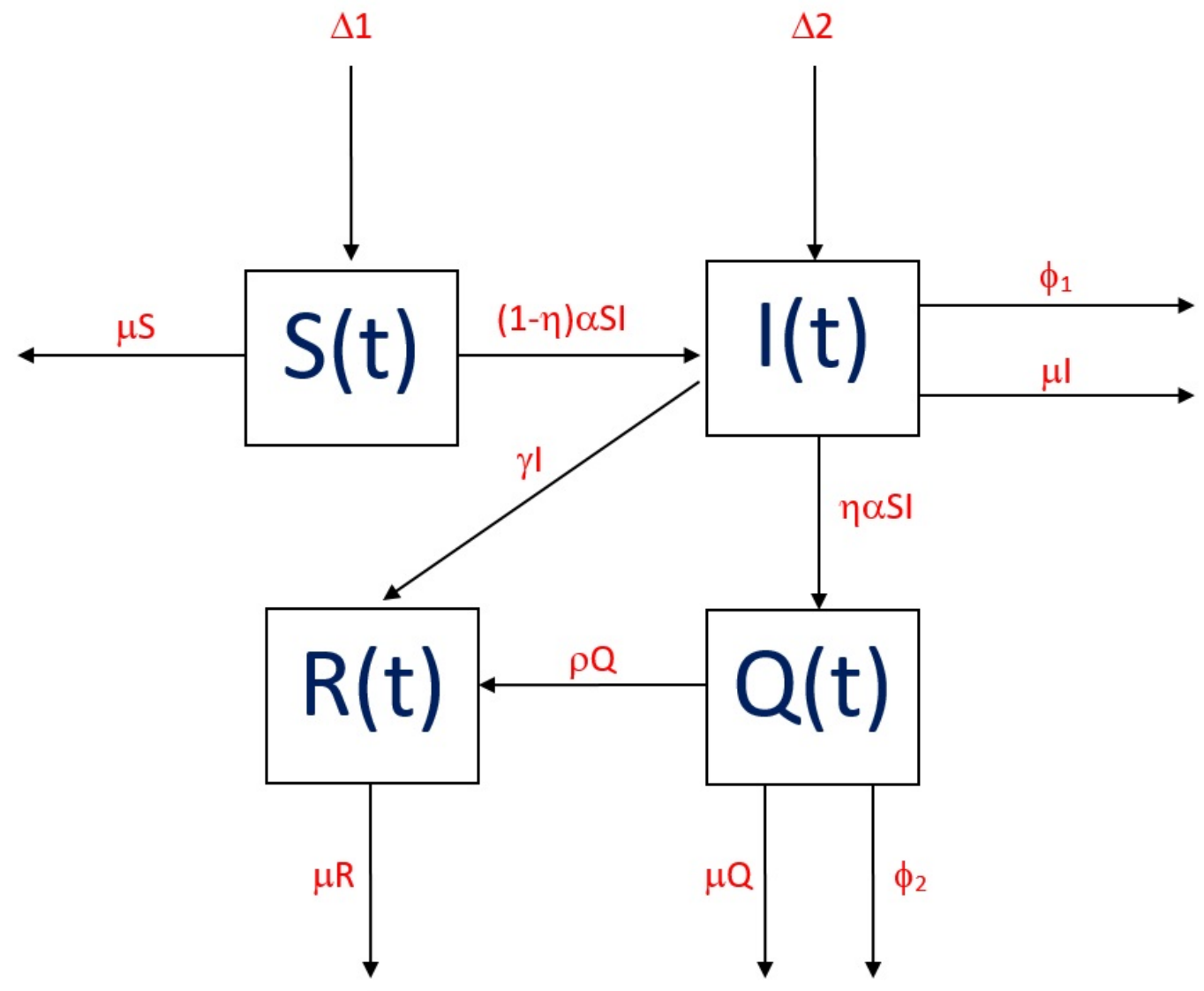

Figure 1. Flow Chart.

individuals. For the sake of simplicity, we have decided to assume that an individual exposed to the virus immediately becomes infectious. Lauer et al. (2020) estimated the incubation period between 2 and 10 days, but there are indications that the ability to infect other individuals already exists during this period as Li et al. (2020a) pointed out and as reported by Wölfel et al. (2020) this ability seems to be higher in the first week after symptoms start. We emphasize that the number of COVID-19 cases is probably much higher than the number of tested and confirmed cases according to Li et al. (2020b).

In turn, the population $Q$ corresponds to the individuals that, due to the confirmation of COVID-19 through a test, or, because of a simple suspicion, were isolated or decided to isolate themselves on their own account (conscious isolation). For the viability of the model, we assume that there is no occurrence of contamination of susceptible individuals by individuals in $Q$ and that the isolation of individuals in this compartment happens immediately after infection. We observe that individuals in compartment $Q$, as well as those in $R$, essentially have no influence on the spread of the disease.

Among the supplementary hypotheses, we assume that the mortality parameter due to infection can be treated with existing international data, thus assuming that mortality is invariant by region. We know that this hypothesis is very relaxed, since mortality is associated with various social and cultural aspects, among others. However, at this stage of the pandemic it is difficult to estimate mortality parameters such as the infection fatality rate, for example, for the local scenario in Manaus 
due to insufficient data. Moreover, our hypothesis on the invariance by region of such parameters will not significantly alter the conclusions of this work. In all compartments (populations) we admit natural death $\mu$. In compartments I and $Q$ we also assume death due to infection (with rates $\varphi_{1}$ and $\varphi_{2}$ respectively). These two rates are used to distinguish between deaths due to infection from group I or from group $Q$.

The transmission takes place according to the incidence rate $\alpha$ laccording to some sources, the force of the infection) and the number of individuals infected per unit of time is determined by alS. These newly infected individuals, move to compartment I of infectious individuals (capable of transmitting disease) at rate $(1-\eta) \alpha / S$ and to the compartment $Q$ of infectious, but isolated individuals (therefore unable to transmit the disease) at rate $\eta \alpha / S$. The total population $N(t)$ naturally satisfies the equality

$$
N(t)=S(t)+I(t)+Q(t)+R(t)
$$

and the model described in Figure 1 corresponds to the following system of ordinary differential equations:

$$
\begin{array}{lr}
\frac{d S}{d t}= & \Delta_{1}-\alpha S I-\mu S \\
\frac{d l}{d t}= & \Delta_{2}+\left(1-\varphi_{1}\right)(1-\eta) \alpha S I-(\gamma+\mu) I \\
\frac{d Q}{d t}= & \left(1-\varphi_{2}\right) \eta \alpha S I-(\rho+\mu) Q \\
\frac{d R}{d t}= & \left(\varphi_{1}(1-\eta)+\varphi_{2} \eta\right) \alpha S I+\rho Q+\gamma l-\mu R .
\end{array}
$$

\section{Estimation of Parameters}

According to Baud et al. (2020), the lethality rate of the disease is estimated at $5.6 \%$ of the confirmed cases in China by March $1^{\text {st }}, 2020$. Furthermore, according to Li et al. (2020b), around $86 \%$ of the cases of the disease were probably undocumented in the period from January $10^{\text {th }}, 2020$ until January $23^{\text {rd }}$, 2020 in China. Thus, we can estimate the lethality rate of infectious individuals at $0.784 \%$.

As a result of this discussion, we assume that at least $14 \%$ of the cases of the disease in Manaus are documented with sufficient speed so that isolation can occur. This way, we will vary the isolation rate $\eta$ in the interval $[0.14,1]$.

For the lethality rate of the population $Q$, we use the following formula, according to the isolation rate, that is, the parameter $\eta$. We calculate

$$
\varphi_{2}=\left(0.056 \cdot 0.14+\varphi_{1} \cdot(\eta-0.14)\right) / \eta
$$

where $0.14 \leq \eta \leq 1$. Thus we observe that the $14 \%$ of infectious individuals, which we assume to be documented, are subject to a fatality rate of 5.6\%. Any number of infectious individuals, which enter the compartment $Q$ in excess of these $14 \%$, for example through conscious isolation, will be assumed to be less severe cases and to be subject to the lower fatality rate of $\varphi_{1}$.

Our estimate of the recovery rate $\rho$ of isolated infectious individuals follows an analogous argument. We estimate the recovery rate to be $\gamma=1 / 14.25$ days $^{-1}$ for patients not requiring 
special care, such as hospitalization based on the study of Chang et al. (2020). For patients in the $Q$ compartment, we estimate the recovery rate with the formula

$$
\rho^{-1}=(1-\eta) \cdot(3.25 / 0.14)+\gamma^{-1}
$$

where $0.14 \leq \eta \leq 1$. Therefore, we observe that the infectious individuals in $Q$ have longer recovery times, depending on $\eta$, that is, on the proportion of tested and confirmed cases (many of which will be severe) in $Q$.

The number of confirmed cases in Manaus by the FVS-AM had a doubling time of 4 to 5 days during the period from March $28^{\text {th }}$ until April $6^{\text {th }}$. This is close to the number of days estimated in the literature: the epidemic doubling time was estimated at 6.4 days by Wu et al. (2020b), at 5 days by Ferretti et al. (2020) and between 4.6 and 6.1 days by Wu et al. (2020a). For the basic scenario (without social distancing and isolation of $14 \%$ of infectious individuals) we choose the parameter $\alpha$ such that the number of infectives has a doubling time of around 4.8 days. According to Hethcote (2000), Section 2.3, p. $605, R_{0}=\alpha /(\gamma+\mu)$. Thus choosing $R_{0}=3.7$ we obtain the desired value for $\alpha$. Moreover, our choice for the value of $R_{0}$ is within the range of estimates $(1.4-4.2)$ that can be found in the literature, cf. Villela (2020) and Choi \& Ki (2020).

For the feasibility of the model, we assume that data of global origin regarding the virus is constant, including that mortality due to infection is invariant by region. This facilitates the estimation of parameters, due to the lack of reliable local data. In our model we disregard the possible occurrence of mutations of the virus SARS-CoV-2.

The parameter $\Delta_{2}$ is assumed to be equal to zero, that is, new individuals enter only the susceptible population and not the infectious one. Thus we are measuring how the population already present behaves, in the presence of some infected individuals. Even if we admitted the insertion of infectious individuals, the number should be very small and would not significantly change the result of the scenarios. For the short term horizon that is the focus of this study, the arrival of infected individuals in Manaus will only have a very limited impact on the evolution of the spread of COVID-19 in the city.

Regarding the initial conditions of the model, we assume a number of 3,379 cases of COVID-19 in Manaus on April $6^{\text {th }}, 2020$. This estimate is based on the fact that there were 473 confirmed cases in Manaus on that date and the estimation that $86 \%$ of cases may not be documented. We assume that the 473 confirmed cases are in the compartment $Q$ and that the 2,906 undocumented cases are in compartment $I$. We further assume that $R=0$ and that the remainder of the population of Manaus is susceptible to the disease. We estimate the population of the municipality of Manaus to be 2,182,763 inhabitants according to data from Instituto Brasileiro de Geografia e Estatística (IBGE 2019) for 2019. Due to the limitations of our model, which does not take into account certain specificities of human behaviour that influence the spread of the virus in the population, see Meyer \& Held (2014), which can lead to a slight overestimation of the number of cases close to the peak of the epidemic, we have decided to work with a number of 1,964,487 inhabitants (90\% of the number mentioned above). A summary of parameter values can be found in Table I. 
Table I. Parameter Values.

\begin{tabular}{|c|c|l|}
\hline Parameter & Value & Comments \\
\hline$\alpha$ & $\zeta R_{0}(\gamma+\mu)$ & We estimate $R_{0}=3.7$, varying the parameter $\zeta$ in the interval [0,1]. \\
\hline$\mu$ & $0.00445 / 365$ & Crude mortality rate in the State of Amazonas* \\
\hline$\gamma$ & $1 / 14.25$ & $\begin{array}{l}\text { We estimate the average recovery time for non-hospitalized infectious } \\
\text { patients at } 14.25 \text { days according to Chang et al. } 2020 .\end{array}$ \\
\hline$\varphi_{1}$ & 0.0034 & see Worldometer 2020 \\
\hline$\varphi_{2}$ & $($ see text) & \\
\hline$\rho$ & $($ see text) & \\
\hline$\eta$ & $0.14 \leq \eta \leq 1$ & This parameter is varied in the scenarios. \\
\hline$\Delta_{1}$ & $0.002016 / 365$ & Crude natality rate in the State of Amazonas* \\
\hline$\Delta_{2}$ & 0 & (see text) \\
\hline IBGE (2019) & &
\end{tabular}

\section{SCENARIOS VARYING THE PARAMETER $\alpha$}

Let $w$ be the number of times an individual comes in contact with others per unit of time and let $p$ be the probability of infection for a single contact. Then $p w$ represents the ability of an infected individual to generate new infectives (infect susceptibles). Assuming that this individual only infects susceptibles, pwS/ $\mathrm{N}$ represents the capacity of this individual to infect other susceptible individuals in this population and the mean number (or rate) of new infectives, per unit of time, can be estimated by $\operatorname{pw}(S / N) I$. If the contact rate is proportional to the population size $N$, say $w=k_{0} N, k_{0} \in \mathbb{R}, k_{0}>0$, then the estimated value or this rate, per unit of time, is $\alpha \mathrm{SI}$, where $\alpha=p k_{0}$ is the transition coefficient, cf. Hethcote 2000. Thus, the variation of $\alpha=p w / N$ corresponds to a change in the number of contacts between people in the susceptible and in the infected compartment. To draw a scenario of social distancing we assume a reduction in the parameter $w$, that is, in the number of times that an individual has contact with other individuals per unit of time.

We estimate the basic reproduction number $R_{0}=3.7$ and we estimate the average time of the infectious period to be 14.25 (see above). In this section we project different social distancing scenarios, where we assume that a percentage $q$ of the population is observing a "stay-at-home" recommendation rigorously and the remainder of the population is continuing daily routine normally. We assume that in a scenario where a "stay-at-home" recommendation is observed, contacts with members of the same household will continue (indeed they will be intensified), but contacts with people outside the household will be strictly reduced. This way, we assume that the ability of individuals to infect members of their own household will not be affected by a "stay-at-home" recommendation and that social distancing thus does not occur on an individual level but occurs "per household".

According to IBGE (2019) the average household in the State of Amazonas is composed of 4 people. We assume that in each household that observes the "stay-at-home" recommendation, all members practice strict isolation, except for one person, who continues to have some interaction 
with the outside world. For the part of the population practising strict isolation, we assume a basic reproduction number of 0.99 , and for members of a household who continue to maintain contact with the outside world, we assume a basic reproduction number of $\left(0.99+R_{0}\right) / 2$. We emphasize that a basic reproduction number of less than 1 is necessary for suppression of the epidemic to occur. In other works, social distancing is modelled through a reduction of the value of $R_{0}$ by a certain factor, subject to a contact matrix, see for example Priem et al. 2020. We consider however our model of the effect of a reduction in mobility to be more realistic for our purposes. Indeed, at least over the short term horizon which is the focus of this work, even infectious individuals practising social distancing will continue to infect other members of their household, thus we assume the value of $R_{0}=0.99$ for this group of people. Furthermore, one member of the household will need maintain contact to the outside world.

Therefore, we vary the parameter $\alpha=\zeta R_{0}(\gamma+\mu)$, with

$$
\zeta=\left(\frac{3 q}{4} \cdot 0.99+\frac{q}{8}\left(0.99+R_{0}\right)+(1-q) \cdot R_{0}\right) / R_{0}
$$

where $0 \leq q \leq 1$

We project four scenarios according to the variation of parameter q: a scenario of limited social distancing, where only a small portion of about $q=20 \%$ of the population are observing a "stay-at-home" recommendation, a scenario of intermediate social distancing, where about half, i.e. $q=50 \%$ of the population are isolating themselves in their homes and a lockdown scenario, where $q=80 \%$ of the population is observing a "stay-at-home" order. This way, we exercise control over the dynamics of the contacts between susceptibles $S(t)$ and infectious $I(t)$ individuals (see Figure 1).

We obtain a growth of case numbers according to Figure 2 over the horizon of 120 days.

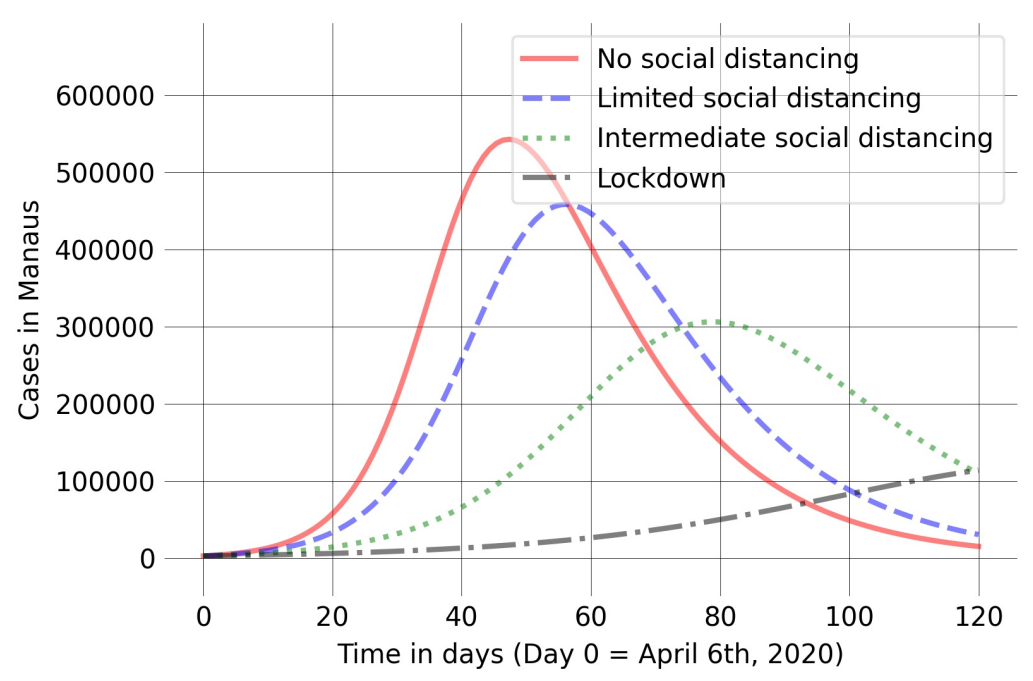

Figure 2. Controlling the contact rate -120 days horizon. 


\section{SCENARIOS VARYING THE PARAMETER $\eta$}

For the analysis of the other scenarios, we varied the parameter $\eta$, i.e. the isolation rate of infectious individuals. We consider that in any scenario $\eta$ is greater than or equal to 0.14 , that is, at least $14 \%$ of infectious individuals are documented and consequently isolated. This figure is based on the number of cases identified in Wuhan in China in January 2020, see subsection "Estimation of Parameters".

This parameter can be interpreted in two ways: as a measure of the amount of tests being performed on the general population or as a measure of a collective awareness among the general population regarding the importance of measures of social distancing. Concerning the first measure, we observe that an increase in $\eta$ corresponds directly to an increase in individuals in the compartment $Q$, that is, in individuals that are strictly isolated - which occurs in the case of a positive test. As a measure of a collective awareness of the importance of social distancing measures, we observe that, according to the system of differential equations above, an increase in $\eta$ directly leads to a smaller growth of the I compartment of infectious individuals capable of spreading the disease.

We emphasize that an increased collective awareness among the general population, can lead to people to decide to isolate themselves in their homes (conscious isolation), avoiding unnecessary circulation and agglomeration. In addition, this awareness may lead people, who have minimal symptoms or whoever had recent contact with a large number of people to naturally decide to isolate themselves at home.

We outline two scenarios that we compared to the basic scenarios in which $\eta=14 \%$, which represents a basic collective awareness - corresponding to the isolation of only confirmed cases via a test): a scenario with an intermediate collective awareness $(\eta=28 \%)$ and a scenario with a raised collective awareness $(\eta=42 \%)$.

When analysing these isolation rates we obtain over a period of 120 days a growth in case numbers as shown in Figure 3.

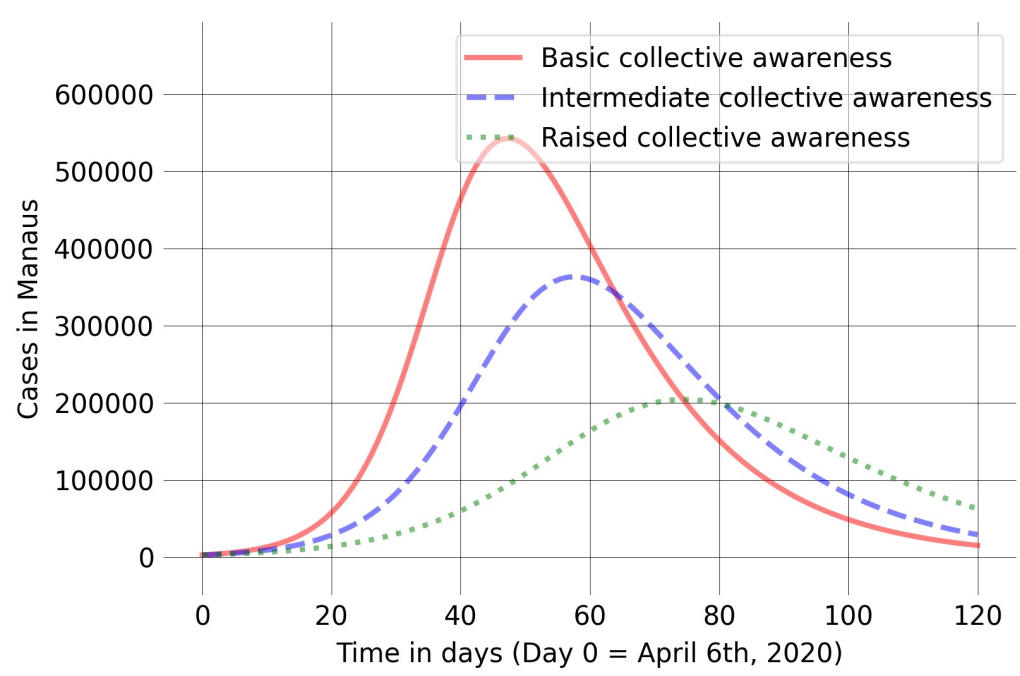

Figure 3. Controlling the isolation rate of infectious individuals - 120 days horizon. 


\section{CONCLUSIONS}

Public policies, in crises caused by epidemics, seek a reduction in the rate of increase of infections to avoid an overload of the health care system. Such an overload occurs in a scenario with an expressive peak of infectious individuals (active cases), all of them simultaneously carriers of the disease. In this work, we analyse two ways to achieve a reduction and a delay of this peak, namely: the isolation of the general population in their homes at different degrees (the population observing a "stay-at-home recommendation") and the increase in the isolation rate of infectious people (through a better identification of these, either with the help of tests or due to better collective awareness of the importance of social distancing measures, especially by individuals with some degree of suspicion of the disease).

Regarding the limitations, we emphasize that our study has only evaluated the consequences of social distancing and isolation scenarios on the number of cases and deaths due to COVID-19 and not the eventual economic or social consequences. Furthermore, given the lack of reliable data at this stage of the epidemic, we opted for simplifications in modelling the flow of individuals between compartments. Also, we assume that the isolation scenarios are perfect, in the sense that there is no contact of isolated individuals with the population in general. We stress that it was possible to analyse lockdown and social distancing scenarios for which there have been concrete proposals formulated - in particular, all isolation scenarios of the population presuppose the isolation of entire households, without the possibility of isolating certain people and not others in the same household. Our projections find limits in the fact that there are no precise way to measure a collective awareness of the importance of social distancing measures. Finally, we emphasize that we work with reduced time horizons, because in the medium and long term the scenarios may suffer intervention by government for the purpose of controlling the epidemic. We consider it probable that there will be interventions with drastic measures when the number of cases or deaths reach a certain level, as has happened in the case of several countries in the last months, such as China, Italy, Spain and United States.

Assuming a population of Manaus of 2,182,763 inhabitants and assuming the existence of 473 confirmed cases in Manaus on April $6^{\text {th }}, 2020$, the projected scenarios lead us to the following conclusions:

We observe that by forcing the reduction of $R_{0}$ (the average number of infections caused by an individual) by means of social distance measures such as "stay-at-home" recommendations, we reduce and we postpone the peak in the number of active cases. In a scenario without any containment measures, our projections point to more than 50,000 active cases, and this mark may be exceeded before the end of April. By increasing the degree of social distance, that is, a higher percentage of the population observing "stay-at-home" recommenations, the occurrence of this number of cases can be gradually postponed to the beginning of the month of June. Only in a scenario of a rigorous and extensive lockdown ("stay-at-home" order), implemented and observed as of March $23^{\text {rd }}, 2020$, would it be possible to have a number of cases less than 50,000 in the first half of June. This can be seen in Figure 2 and in Table II.

We emphasize that, according to our projections, an amount of around 50,000 active cases of COVID-19 would result in at least 1,000 deaths, with hundreds of these occurring on the same day. 
Table II. Social Distancing - number of cases (Day o $=$ April $6^{\text {th }}, 2020$ ).

\begin{tabular}{|r|r|r|r|r|}
\hline \multicolumn{5}{|c|}{ Social Distancing } \\
\hline Horizon & None & Limited & Intermediate & Lockdown \\
\hline 15 days & 25,00 & 17,000 & 9,000 & 5,000 \\
\hline 30 days & $>50,000$ & $>50,000$ & 30,000 & 9,000 \\
\hline 60 days & $>>50,000$ & $>>50,000$ & $>50,000$ & 26,000 \\
\hline
\end{tabular}

Only in the scenario of extensive and rigorous social distancing in the form of a lockdown will deaths occur more gradually.

An increase in the number of isolated infectious individuals, that is, an increase in the parameter $\eta$, can be achieved by substantially increasing the number of tests performed, i.e. not just testing symptomatic people or individuals but also individuals with high probability of having the disease, according to different considerations. A conceivable way would be the strict isolation of individuals who have had contact with a large number of people in recent days or who have some mild symptoms that may be associated with COVID-19, or strictly limiting the number of people an individual may have contact in a certain time period. Regarding the symptoms, it is noteworthy that some of these are similar to those of influenza according to Center for Disease Control and Prevention, however there are indications, as reported by Royal College of Surgeons of England (2020), that there are others, more specific of the disease COVID-19, such as the loss of smell for example. In order to obtain a better efficiency in isolating infectious individuals, that is, an increase in the parameter $\eta$, it may be recommended that individuals with such a symptom (or with other symptoms that may be associated with COVID-19) isolate themselves spontaneously.

Regarding the scenarios with greater efficiency in the isolation of infectious, we observe that with a slightly increased collective awareness (increasing the number of isolated infectious individuals through tests or through better awareness of the population) 50,000 cases will be exceeded in early May. In a scenario with a more significant collective awareness, there will be 50,000 cases of the disease a little later, in mid-May. This can be seen in Figure 3 and Table III.

Table III. Collective Awareness - number of cases (Day o $=$ April $6^{\text {th }}, 2020$ ).

\begin{tabular}{|r|c|r|r|}
\hline \multicolumn{4}{|c|}{ Collective Awareness } \\
\hline Horizon & Basic & Intermediate & \multicolumn{1}{|c|}{ Raised } \\
\hline 15 days & 25,000 & 15,000 & 9,000 \\
\hline 30 days & $>50,000$ & $>50,000$ & 28,000 \\
\hline 60 days & $>>50,000$ & $>>50,000$ & $>50,000$ \\
\hline
\end{tabular}

We observe that given the current level of the number of cases, an increase in the parameter $\eta$, to the different levels studied, will not have a great effect on the reduction of the number of cases in the following weeks. Indeed, in no configuration will we be able to remain below the mark of 50,000 
active infections over the horizon of 60 days. However, with a collective awareness of the population adequate to the need of the moment, when the level of cases was still low, or with the execution of a large number of tests at that stage, our simulations reveal that the scenarios of increased collective awareness would have a better effect on postponing the increase in case numbers.

We draw attention to the fact that mitigation measures can lead to a temporary reduction of the number of infections, which can increase again once such measures are reduced or eliminated (due to a still large number of susceptible individuals). Therefore, although the present study is specific to the scenario of the pandemic in Manaus in the month of April 2020, mitigation efforts may reduce cases to a level so that the conclusions of this study, concerning social distancing and an awareness of the general public regarding the need to practice conscious and voluntary isolation, may be of use to policy makers at a future point in time.

\section{ACKNOWLEDGMENTS}

The authors would like to thank the referees for their careful reading and useful comments which improved the paper. The authors are partially supported by Conselho Nacional de Desenvolvimento Científico e Tecnológico (CNPq) of the Ministério da Ciência, Tecnologia e Inovação (MCTI - Brazilian Ministry of Science, Technology and Innovation).

\section{REFERENCES}

BAO L \& OTHERS. 2020. Reinfection could not occur in SARS-COV-2 infected rhesus macaques. doi:10.1101/2020.03.13.990226.

BAUD D, QI X, NIELSEN-SAINES K, MUSSO D, POMAR L \& FAVRE G. 2020. Real estimates of mortality following COVID-19 infection. The Lancet Infectious Diseases doi:10.1016/S14733099(20)30195-X.

CASTILHO C, GONDIM J, MARCHESIN M \& SABETI M. 2020. Assessing the Efficiency of Different Control Strategies for the Coronavirus (COVID-19) Epidemic. Preprint. arXiv:2004.03539.

CHANG D, MO G, YUAN X, TAO Y, PENG X, WANG F, XIE L, SHARMA L, CRUZ C \& QIN E. 2020. Time Kinetics of Viral Clearance and Resolution of Symptoms in Novel Coronavirus Infection. Am J Respir Crit Care Med 201(9): doi:10.1164/rccm.202003-0524LE.

CHOI S \& KI M. 202O. Estimating the reproductive number and the outbreak size of COVID-19 in Korea. Epidemiology and Health 42. doi:10.4178/epih.e2020011. Published online: March 12, 2020.

CONTROL CFD \& PREVENTION. 2020. https://www.cdc.gov/ coronavirus/2019-ncov/symptoms-testing/symptoms.html.

FERRETTI L, WYMANT C, KENDALL M, ZHAO L, NURTAY A, ABELER-DÖRNER L, PARKER M, BONSALL D \& FRASER C. 2020. Quantifying SARS-CoV-2 transmission suggests epidemic control with digital contact tracing. Science 368(6491): doi:10.1126/science.abb6936.
FVS AM - FUNDAÇÃO DE VIGILÂNCIA EM SAÚDE DO ESTADO DO AMAZONAS. 202O. http://www.fvs.am.gov.br/. Accessed on April $6^{\text {th }}$.

GOVERNOR OF THE STATE OF AMAZONAS. 2020. https: / /amazonasatual.com.br/wp-content/uploads/2020/03/ DECRETO-N.-42.101-DE-23-DE-MARCl\%CCl\%A7O-DE-2020.pdf. Decree N. 42.101 of March $23^{\text {rd }}$.

HETHCOTE HW. 2000. The mathematics of infectious diseases. SIAM Review 42(4): 599-653.

IBGE - INSTITUTO BRASILEIRO DE GEOGRAFIA E ESTATÍSTICA. 2020. https://cidades.ibge.gov.br/brasil/am/manaus/panorama. https:// biblioteca.ibge.gov.br/visualizacao/livros/liv45700.pdf. Accessed on April $6^{\text {th }}, 2020$.

IMPERIAL COLLEGE LONDON, UK. 2020. COVID-19 reports. https://www.imperial.ac.uk/mrc-globalinfectious-diseaseanalysis/covid-19/. Accessed on April $6^{\text {th }}, 2020$.

LAUER S, GRANTZ K, BI Q, JONES F, ZHENG Q, MEREDITH H, AZMAN A, REICH N \& LESSLER J. 2020. The Incubation Period of Coronavirus Disease 2019 (COVID-19) From Publicly Reported Confirmed Cases: Estimation and Application. Ann Intern Med 172(9): 577-582.

LI P ET AL. 2020a. Transmission of COVID-19 in the terminal stage of incubation period: a familial cluster. Int J Infect Dis 96: 452-453.

LI R, PEI S, CHEN B, SONG Y, ZHANG T, YANG W \& SHAMAN J. 202ob. Substantial undocumented infection facilitates the 
rapid dissemination of novel coronavirus (SARS-CoV2. Science 368(6490): 489-493.

MEYER S \& HELD L. 2014. Power-Law Models For Infectious Disease Spread. Ann Appl Stat 8: 1612-1639.

PREM K, LIU Y, RUSSELL T, KUCHARSKI A, EGGO R \& DAVIES N. 2020. The Effect of Control Strategies to Reduce Social Mixing on Outcomes of the COVID-19 Epidemic in Wuhan, China: A Modelling Study. The Lancet Public Health 5(5): doi:10.1016/S2468-2667(20)30073-6.

ROCHA FILHO T, DOS SANTOS F, GOMES V, ROCHA T, CRODA J, RAMALHO W \& DE ARAÚJO W. 2020. Expected impact of COVID-19 outbreak in a major metropolitan area in Brazil. doi:10.1101/2020.03.14.20035873.

THE ROYAL COLLEGE OF SURGEONS, UNITED KINGDOM. 2020. https:/ / www.entuk.org/loss-sense-smell-marker-covid-19infection. Accessed on April $6^{\text {th }}, 2020$.

VILLELA DAM. 2020. The value of mitigating epidemic peaks of COVID-19 for more effective public health responses. Revista da Sociedade Brasileira de Medicina Tropical 53: 1-2.

WHO - WORLD HEALTH ORGANIZATION. 2020. Director-General's opening remarks at the media briefing on COVID-19. https://www.who.int/dg/speeches/detail/who-director-

general-s-openingremarks-at-the-media-briefing-on-covid-1911-march-2020. Accessed on April $6^{\text {th }}, 2020$.

WOLFEL R ET AL. 2020. Clinical presentation and virological assessment of hospitalized cases of coronavirus disease 2019 in a travel-associated transmission cluster. doi:10.1101/2020.03.05.20030502.

WORLDOMETERS. 2020. http://worldometers.info/coronavirus. Accessed on April $6^{\text {th }}, 2020$.

WU J, LEUNG K, BUSHMAN M, KISHORE N, NIEHUS R, SALAZAR P, COWLING B, LIPSITCH M \& LEUNG G. 2020a. Estimating clinical severity of COVID-19 from the transmission dynamics in Wuhan, China. Nature Medicine 26: 506-510.

WU J, LEUNG K \& LEUNG G. 2020b. Nowcasting and forecasting the potential domestic and international spread of the 2019-nCoV outbreak originating in Wuhan, China: a modelling study. The Lancet 395(10225): 689-697.

\section{How to cite}

BITAR S \& STEINMETZ WA. 2020. Scenarios for the Spread of COVID-19 in Manaus, Northern Brazil. An Acad Bras Cienc 92: e20200615. DOI 10.1590/0001-3765202020200615.

Manuscript received on April 24, 2020;

accepted for publication on June 4, 2020

SANDRO BITAR

https://orcid.org/0000-0001-6971-2477

WILHELM ALEXANDER STEINMETZ

https://orcid.org/0000-0002-9314-4999

Departamento de Matemática, Universidade Federal do Amazonas, Av. General Rodrigo Octávio, 6200, 69080-900 Manaus, AM, Brazil

Correspondence to: Wilhelm Alexander Steinmetz

E-mail:was@ufam.edu.br

\section{Authors contributions}

The two authors contributed equally to this work.

\section{(cc) BY}

